\title{
New Diagnostic Tests and New Therapies for Glomerular Diseases
}

\author{
Gerald B. Appel Alice Sue Appel \\ Glomerular Kidney Disease Center, Division of Nephrology, Columbia University Medical Center, and College of \\ Physicians and Surgeons, Columbia University, New York, N.Y., USA
}

\section{Key Words}

Focal segmental glomerulosclerosis - Membranous

nephropathy $\cdot \lg \mathrm{A}$ nephropathy $\cdot \mathrm{C} 3$ glomerulopathies

\begin{abstract}
There has recently been considerable progress in our understanding of the pathology and mechanisms of a number of primary glomerulopathies. However, without parallel advancement in both our diagnostic skills and treatment methods, such advances cannot be translated into clinical progress. Fortunately, a number of new promising diagnostic tests are being evaluated in patients with a variety of primary glomerulopathies including focal segmental glomerulosclerosis membranous nephropathy, IgA nephropathy, and C3 glomerulopathies. Likewise, a number of new therapeutic interventions are available to treat these entities, including the pituitary hormone $\mathrm{ACTH}$, the monoclonal antibody rituximab, and the monoclonal blocker of the fifth component of complement, eculizumab. All three are already FDA-approved for at least one glomerular disease. This article will discuss these newer diagnostic tests and therapies as well as their potential roles in patients with glomerular diseases and where they fit in with the rest of our therapeutic armamentarium in treating patients with glomerular diseases.

Copyright $\odot 2013$ S. Karger AG, Basel
\end{abstract}

(C) 2013 S. Karger AG, Basel

$0253-5068 / 13 / 0353-0081 \$ 38.00 / 0$

Fax +4161306 1234

E-Mail karger@karger.ch

www.karger.com
Accessible online at:

www.karger.com/bpu

\section{Focal Segmental Glomerulosclerosis}

Focal segmental glomerulosclerosis (FSGS) is a common pattern of glomerular disease occurring in up to 20 $25 \%$ of adults with idiopathic nephrotic syndrome and having an increased incidence in African-Americans [1]. Many patients with FSGS have secondary forms of the disease due to genetic abnormalities of the visceral epithelial cells, viral disease (HIV and parvovirus), medications (heroin and pamidronate), and hyperfiltration in kidneys with reduced nephron mass and 'work overload' (obesity, sickle cell disease, remnant kidneys, etc.) [1]. In most patients, FSGS is idiopathic and associated with large amounts of proteinuria and the nephrotic syndrome with hypoalbuminemia, and edema. Two new areas of research may lead to potential diagnostic tests which in the future may help us both diagnose and prognosticate in patients with FSGS. The first is a genetic test for apolipoprotein L1 (ApoL1) and the second, a test for circulating soluble urokinase receptor (suPar).

Two independent variants in the gene coding for ApoL1, a lipid-binding protein found in serum, have been found to be strongly associated with a higher incidence of FSGS in African-Americans than in Caucasians [2]. The analysis for the presence of such genetic variables is now available through research laboratories. These gene variants are not uncommon in the Black population but ab-

Dr. G.B. Appel

Room 4124, Presbyterian Hospital

622 West 168th Street

New York, NY 10032 (USA)

E-Mailgba2@columbia.edu 
sent in the genes of non-Blacks [3]. In addition, it was found in vitro that the doubly mutated APOL1 gene lysed Trypanosoma brucei rhodesiense, the organism that can cause African sleeping sickness. Thus, in the evolutionary process, the mutations of the APOL1 gene could have resulted in protection from African sleeping sickness in areas where trypanisomiasis is endemic. Studies suggest that the presence of these G1 and G2 alleles for ApoL1 are associated with sclerosing glomerulopathies such as FSGS and HIV-associated nephropathy and hypertensive risk in African-Americans [4]. The findings of the genotype of individuals with FSGS hopefully will improve prognostication and treatment regimens for those with disease associated with APOL1 mutations $[2,4]$.

The circulating form of uPAR, the podocyte-bound receptor for urokinase, is suPar. In healthy individuals, suPAR levels are generally low $[5,6]$. In a recent study, it was observed that two thirds of patients with primary FSGS exhibited levels of suPAR significantly higher than in healthy individuals or in others with different proteinuric diseases of the glomeruli [including membranous nephropathy $(\mathrm{MN})$, minimal change disease in remission or relapse, and preeclampsia] $[5,6]$. It is also well known that FSGS can recur in up to $30 \%$ of patients receiving a renal transplant [1]. This fact has suggested the presence of a circulating 'permeability factor' or factors associated with FSGS, causing the reappearance of the disease in the transplanted, previously healthy kidney. Increased levels of suPAR are also found in individuals who have experienced recurrence of FSGS in a transplant [5].

It is hypothesized that in FSGS the loss of the integrity of the podocyte cytoskeleton allows major protein loss; hence the nephrotic syndrome results from the presence of abnormal amounts of suPAR. In vitro and animal data suggest foot process effacement in FSGS may result from activation of podocyte $\beta_{3}$-integrin by suPAR with the alteration of the podocyte cytoskeleton. Serum assays for suPAR may in the future help diagnose FSGS in nephrotic patients. If, indeed, suPAR does cause idiopathic FSGS, newer modes of therapy can be anticipated. In addition to plasmapheresis to lower the concentration of suPAR, medications may be developed to inhibit the interaction of suPAR with $\beta_{3}$-integrin, thus negating the influence of the glomerular permeability in FSGS.

Typical patients with untreated primary FSGS do not have a spontaneous remission of their nephrotic syndrome and most progress to renal failure and dialysis over 5-20 years from presentation [1]. Although untested against other agents in adults with FSGS, high-dose corticosteroids either daily or every other day with a ta- pering dose are considered standard first-line therapy. Therapy must be continued for 4-6 months before the patient is considered resistant to corticosteroids. For those patients with steroid-resistant disease (approx. one half and higher in Blacks), cyclosporine has proven effective in inducing remissions of the nephrotic syndrome and preserving renal function over time in a double-blind controlled randomized trial [7]. Mycophenolate, cyclophosphamide, and tacrolimus have also been used in a variety of other studies. Two new agents that have been used here are rituximab and ACTH, each with varying results.

Rituximab, a chimeric murine-human monoclonal antibody against the CD20 antigen of B cells, rapidly depletes circulating $\mathrm{B}$ cells [8]. The drug has been FDA-approved for several diseases including certain non-Hodgkin's lymphomas and moderate to severe rheumatoid arthritis. Recently, after success in randomized controlled trials, it was approved for ANCA-positive small vessel vasculitis and glomerulonephritis [9]. Since rituximab is a partial murine chimeric molecule, it must be infused slowly and should be given with enough corticosteroids to prevent severe allergic attacks. While controlled randomized trials have only been performed in ANCA-positive vasculitis and lupus nephritis, the drug has, however, been extensively used in a variety of other glomerular diseases in uncontrolled trials [8-10].

Rituximab has been used in small trials of nephrotic patients with FSGS. In several of these studies, some patients with steroid-resistant nephrotic syndrome or biopsy-documented FSGS have responded to this therapy. In corticosteroid-dependent or relapsing nephrotics the drug has led to reduction of proteinuria and the ability to decrease other immunosuppressive agents. Recently, however, results have been disappointing in corticosteroid-resistant patients [11]. A Spanish collaborative group found that of 8 patients with steroid-resistant FSGS, only 3 responded and even they needed a repeat course of the drug. A more recent controlled trial in steroid-resistant FSGS found no benefit in the addition of rituximab in terms of remissions or reducing other immunosuppressives [11]. Thus, this agent has been somewhat disappointing so far in treating resistant FSGS.

Although listed here as a new therapy for glomerular diseases, the natural pituitary hormone ACTH has been FDA-approved for treatment of the nephrotic syndrome since the 1950s [12]. Its resurgence has come as a result of the successful use in Europe of synthetic ACTH, currently unavailable in the USA, in treating the nephrotic syndrome [13]. In some such studies, several patients with 
FSGS had dramatic decreases in proteinuria after treatment with ACTH.

In subsequent publications some patients resistant to other therapies have responded well to natural ACTH treatment $[14,15]$. Two recent studies looked specifically at treatment with ACTH in resistant FSGS and found promising results $[16,17]$. In one from Stanford University, proteinuria was significantly reduced in a group of 12 FSGS patients. In the other study, from Columbia University, 12 FSGS patients who had failed on average three immunosuppressive regimens (e.g. corticosteroids, CNI, mycophenolate, etc.) were treated with Acthar gel 80 units twice weekly for 6 months. Five of these patients had a solid response to treatment with major sustained drops in proteinuria and/or reduction of serum creatinines. Thus, ACTH may be a viable therapy for resistant FSGS patients.

\section{Membranous Nephropathy}

$\mathrm{MN}$ remains the most common pattern of idiopathic nephrotic syndrome in Caucasian patients and hence in the USA [18]. While MN is a pattern of glomerular damage that may be associated with infectious etiologies, systemic lupus erythematosus, medications, and with certain tumors, most cases are still primary or idiopathic and present with full-blown nephrotic syndrome [18]. However, some secondary forms of the disease present with proteinuria and the nephrotic syndrome before it is clear they are secondary to lupus, medications, or an underlying malignancy. This has led to multiple serologic and other testing to exclude secondary forms of the disease before treatment is instituted. In patients over 65 years of age, extensive searches for underlying tumors can include CT scans of the chest, upper gastrointestinal series, colonoscopy, gynecologic and prostate examinations, all of which are expensive and time-consuming, thus delaying immunosuppressive therapy. Discovering a serologic test to classify patients into primary diopathic and secondary forms of $\mathrm{MN}$ would be a huge benefit to clinicians and their patients.

In many cases of idiopathic MN, the M-type phospholipase-2 receptor has been shown to be the antigen in the subepithelial electron-dense immune deposits [19]. Circulating antibodies to this receptor have been found in $70-80 \%$ of patients with idiopathic MN. They have been absent in secondary forms of the disease since other antigens and antibodies against them are involved in their pathogenesis. During successful treatment of $\mathrm{MN}$ with immunosuppressive agents the titer of the antibody falls [20]. In the future, evaluation of the nephrotic patient may include not only an ANA, anti-DNA, ANCA and other serologic tests, but measurement of the antibody to the M-type phospholipase receptor. A high positive test for this circulating antibody will predict idiopathic disease and hopefully preclude extensive evaluations for other causes of MN. When the patient's urinary protein level declines, absence of the antibody will predict an immunologic response to therapy rather than just a hemodynamic response perhaps due to ACE inhibition or $\mathrm{ARB}$ use.

In $\mathrm{MN}$ renal survival is usually more than $75 \%$ at 10 and there is a spontaneous remission rate of between 20 and $30 \%$. In general, older patients, males, and those with heavy persistent proteinuria are most likely to progress to renal failure and hence to benefit from therapy. A number of therapies have proven effective in randomized controlled trials. As first-line therapy, KDIGO guidelines recommend alternating months of corticosteroid therapy and oral cytotoxic therapy (either cyclophosphamide or chlorambucil) over 6 months attempting to achieve both total remissions and preservation of renal function [18]. Calcineurin inhibitors, cyclosporine and tacrolimus have given increased remissions of the nephrotic syndrome and improved renal survival as well in controlled trials. Mycophenolate has been successful in inducing remission when compared to retrospective use of cytotoxics, but has not been studied in a large randomized trial. Despite these multiple therapeutic options, some patients with compelling indications for therapy fail to respond and need alternative therapy. Rituximab and ACTH have both been used in this situation.

Rituximab has proven effective in many nonrandomized trials of $\mathrm{MN}$ patients [21, 22]. In most of the trials the mean proteinuria has fallen significantly. A recent study by Ruggenenti et al. [23] looked at 100 patients treated with rituximab for idiopathic MN. 27\% of the patients had a complete and $38 \%$ a partial remission of the nephrotic syndrome. The authors feel this may be a firstline agent for some patients. However, in this study, two thirds of patients had received no prior treatment for their MN [24]. Moreover, this was not a randomized controlled trial. Nevertheless, it is clear many patients will respond to this therapy.

Synthetic ACTH has been studied in both anecdotal series and a randomized controlled trial in idiopathic $\mathrm{MN}[12-14,24]$. In a controlled randomized trial by Ponticelli et al. [25] in nephrotic idiopathic $\mathrm{MN}$, patients were 
randomized to receive a standard regimen of alternating months of corticosteroid and an alkylating agent (cyclophosphamide) or ACTH. Patients were matched for degree of proteinuria, GFR, and histology. At the end of 6 months of therapy the group receiving the standard regimen had one third of patients in complete remission and one third in partial remission and one third treatment failures. The ACTH group had one half of patients in complete remission, over one third in partial remission, and only 2 treatment failures. Side effects were mild in both groups. While these studies were done with longacting synthetic ACTH in Europe, several recent trials confirm the efficacy of natural ACTH in the USA. In one study for example, 7 of 10 patients with resistant $\mathrm{MN}$, many of whom failed 2-3 treatment regimens, responded to therapy $[14,15]$. Likewise, in a recent abstract, 10 of 17 resistant $\mathrm{MN}$ patients had a remission of proteinuria (ISN 2011 in Vancouver). Again, natural ACTH has not been studied in a randomized controlled fashion. Fortunately, both rituximab and $\mathrm{ACTH}$, which appear so promising in resistant $\mathrm{MN}$, are currently being evaluated in controlled randomized trials.

\section{IgA Nephropathy}

IgA nephropathy (IgAN) is the most frequent form of idiopathic glomerulonephritis worldwide (comprising $15-40 \%$ of primary glomerulonephritides in parts of Europe and Asia) [26]. A significant percent of patients will progress to ESRD over 20 years, and probably more at 30 and 40 years of follow-up. The diagnosis of IgAN is established purely by the pathology, with the finding of glomerular IgA deposits either as the dominant or codominant immunoglobulin on immunofluorescence microscopy. At present there is no diagnostic clinical test for IgAN and older tests such as amount of the circulating IgA level and IgA fibronectin aggregates have not stood the test of time.

In IgAN, recent studies strongly suggest that most patients have an abnormal circulating IgA immunoglobu$\operatorname{lin}[26,27]$. This IgA is deficient in galactose residues at the hinge region of the immunoglobulin. This is true in diverse populations in both China and the USA, in adult and pediatric populations, and in Henoch-Schönlein purpura as well as in IgAN. Relatives of patients with IgAN also appear to have the same circulating galactosedeficient IgA. There is evidence that the presence of an abnormal IgA molecule is the first of a two-hit process that leads to the inflammatory glomerular lesions of
IgAN. A second hit may be oxidative stress in association with deposited abnormal IgA [27]. Other recent data suggest patients have high levels of circulating IgG and IgA glycan-specific autoantibodies that can form immune complexes with galactose-deficient IgA and lead to immune deposits and incite damage in the kidney [28]. Regardless, in some patients, detection of an abnormal IgA immunoglobulin could lead to a clinical diagnostic test. In the patient with isolated glomerular hematuria where the differential diagnosis includes IgAN, thin basement membrane disease and other disorders of type IV collagen in the glomeruli, a positive test would suggest the diagnosis of IgAN. Also the degree and location of the deficient galactosylation of the IgA immunoglobulin may prove to be prognostic in some patient groups. Likewise, the presence and amount of glycan specific autoantibodies may predict the course of IgA patients.

Multiple therapies have been utilized to treat patients with progressive IgAN or with IgAN with heavy proteinuria. There are now several controlled, randomized trials documenting the efficacy of corticosteroid therapy in patients with over $1 \mathrm{~g}$ proteinuria daily. Rituximab has been used in only a few patients with IgAN and is being studied currently versus placebo for progressive disease. ACTH likewise has also been used in a small number of patients with mixed results $[14,15]$. Neither should replace a trial of corticosteroids in patients likely to have progressive disease.

\section{C3 Glomerulopathies}

Recent studies have revealed that many patients formerly thought to have idiopathic membranoproliferative GN or postinfectious GN, actually have abnormalities of the alternate complement pathway [29]. These may be inherited genetic defects in inhibitors of the alternate complement cascade or antibodies against these inhibitors. The result is one of a number of forms of glomerulonephritis with varying light microscopy but invariable C3 deposition in the glomerulus. The two most common of these disorders are $\mathrm{C} 3$ glomerulonephritis and dense deposit disease [30]. When confronted with these disorders the clinician must now analyze in-depth complement components, their genetics, and also search for antibodies to the inhibitors of this system, factor $\mathrm{H}$, factor I and MCP $[29,30]$. This discovery of new mechanisms of disease with persistent activation of the alternate complement pathway and low $\mathrm{C} 3$ values has led to exciting new 
trials with a humanized monoclonal blocker of the fifth component of complement, eculizumab. Eculizumab has recently been FDA-approved to treat atypical hemolytic uremic syndrome, a hemolytic disorder with glomerular damage mediated by alternate complement pathway activation. Studies in C3 glomerulopathies have used eculizumab for over a year in a number of patients [31, 32]. Preliminary data is promising and may have implications for many other diseases with complement activation as a component.
There has been major progress in our understanding of the mechanisms of a number of glomerular diseases. Fortunately, this progress has been accompanied with some exciting new potential diagnostic tests. While they are not yet in clinical usage, many are available through research laboratories already. Likewise, the development of entirely new types of therapeutic agents to treat these diseases is being studied in controlled randomized trials.

\section{References}

1 D’Agati VD, Kaskel FJ, Falk RJ: Focal segmental glomerulosclerosis. N Engl J Med 2011;365:2398-2341.

-2 Genovese G, Tonna SJ, Knob AU, Appel GB, et al: A risk allele for focal segmental glomerulosclerosis in African-Americans is located within a region containing APOL1 and MYH9. Kidney Int 2010;78:698-704.

$\checkmark 3$ Genovese G, Friedman DJ, Ross MD, et al: Association of trypanolytic ApoL1 variants with kidney disease in African-Americans. Science 2010;329:841-845.

$\checkmark 4$ Reeeves-Daniel AM, DePalma JA, Bleyer AJ, et al: The APOL1 gene and allograft survival after kidney transplantation. Am J Transplant 2011;11:1025-1030.

5 Wei C, El Hini S, Li J, et al: Circulating urokinase receptor as a cause of focal segmental glomeruloslcerosis. Nat Med 2011;17, 952 960.

6 Shankland S, Pollak M: A suPar circulating permeability factor causes kidney disease. Nat Med 2011;17:926-927.

7 KDIGO: Clinical practice guidelines for glomerulonephritis. Kidney Int 2012(suppl 2):186-197.

$>8$ Vincenti F, Cohen SD, Appel GB: Novel B cell therapeutic targets in transplantation and immune-mediated glomerular diseases. Clin J Am Soc Nephrol 2012;5:142-151.

$\checkmark 9$ Stone JH, Merkel PA, Spiera R, et al: Rituximab versus cyclophosphamide for ANCAassociated vasculitis. N Engl J Med 2010;363: 221-232.

-10 Rovin B, Richard Furie R, Latinis K, et al: Efficacy and safety of rituximab in patients with active proliferative lupus nephritis: results from the randomized, double-blind phase III LUpus Nephritis Assessment with Rituximab (LUNAR) Study. Arthritis Rheum 2012;64:1215-1216.

11 Magnasco A, Ravani P, Edefonti P: Rituximab in children with resistant idiopathic nephrotic syndrome. J Am Soc Nephrol 2012;23:1117-1124.
12 Gong R: The renaissance of corticotrophin therapy in proteinuric nephropathies. Nat Rev Nephrol 2011;8:122-128.

13 Berg AL, Arnadottir M: ACTH-induced improvement in the nephrotic syndrome in patients with a variety of diagnoses. Nephrol Dial Transplant 2004;19:1305-1307.

-14 Bomback AS, Tumlin JA, Baranski J, et al: Treatment of nephrotic syndrome with adrenocorticotrophic hormone gel. Drug Design Devel Ther 2011;5:147-153.

15 Bomback AS, Canetta PA, Beck LH, et al: Treatment of resistant glomerular disease with adrenocorticotropic hormone gel: a prospective trial. Am J Nephrol 2012;36:5867.

16 Lafayette R, Root C, Mehta K: Adrenocorticotropin (ACTH) gel for the treatment of primary focal glomeruloslcerosis (FSGS). Abstr Natl Kidney Found, 2012.

17 Hogan JJ, Bomback AS, Canetta PA, et al Treatment of resistant primary focal segmental glomerulosclerosis with ACTH gel. Abstr Natl Kidney Found, 2012.

18 KDIGO Clinical Practice Guidelines for Glomerulonephritis. Kidney Int Suppl 2012; 2:186-197.

19 Beck LH Jr, Bonegio RG, Lambeau G, et al: M-type phospholipase $A_{2}$ receptor as target antigen in idiopathic membranous nephropathy. N Engl J Med 2009;361:11-21.

20 Beck LH Jr, Fervenza F, Beck DM, et al: Rituximab-induced depletion of antiPLA2R autoantibodies predicts response in membranous nephropathy. J Am Soc Nephrol 2011;22:1543-1550.

21 Cravedi P, Sghirlanzoni MC, Marasa M, et al: Efficacy and safety of rituximab second-line therapy for membranous nephropathy: a prospective matched-cohort study. Am J Nephrol 2011;33:461-468.

22 Fervenza FC, Abraham RS, Erickson SB, et al: Rituximab therapy in idiopathic membranous nephropathy: a 2-year study. Clin J Am Soc Nephrol 2010;5:2188-2198.
23 Ruggenenti P, Crevedi P, Chianca A, et al: Rituximab in idiopathic membranous glomerulonephritis. J Am Soc Nephrol 2012;23: 1416-1425.

24 Appel GB: Rituximab in membranous nephropathy: is it a first-line treatment? J Am Soc Nephrol 2012;23:1280-1282.

25 Ponticelli C, Passerini P, Salvadori M, et al: A randomized pilot trial comparing methylprednisolone plus a cytotoxic agent versus synthetic adrenocorticotropic hormone in idiopathic membranous nephropathy. Am J Kidney Dis 2006;47:233-240.

26 Coppo R, Fehally J, Glassock RJ: IgA nephropathy at two score and one. Kidney Int 2010;77:181-186.

27 Camilla R, Suzuki H, Dapra V, et al: Oxidative stress and galactose-deficient IgA1 as markers of progression in IgA nephropathy. Clin J Am Soc Nephrol 2011;6:1903-1911.

28 Berthoux F, Suzuki H, Thibaudin L, et al: Autoantibodies targeting galactose-deficient IgA1 associate with progression of IgA nephropathy. J Am Soc Nephrol 2012;23: 1579-1587.

29 Sethi S, Nestor CM, Smith RJ: Membranoproliferative glomerulonephrtis and C3 glomerulopathy: resolving the confusion. Kidney Int 2012;81:434-441.

30 Sethi S, Fervenza F, Zhang Y, et al: C3 glomerulonephritis: clinicopathological findings, complement abnormalities, glomerular proteomic profile, treatment, and follow-up. Kidney Int 2012;82:465-473.

-31 Herlitz L, Bomback AS, Markowitz GS, Stokes MB, Smith RN, Colvin RB, Appel GB, D'Agati VD: Pathology after eculizumab treatment for dense deposit disease and C3 Glomerulonephritis. J Am Soc Nephrol 2012;23:1229-1237.

>32 Bomback AS, Smith RJ, Barile GR, et al: Eculizumab for the treatment of dense deposit disease and C3 Glomerulonephritis. Clin J Am Soc Nephrol 2012;7:748-756. 